\title{
Surgery impacts right atrial function in tetralogy of Fallot
}

\author{
Eugenie Riesenkampff, MD, ${ }^{\mathrm{a}}$ Nadya Al-Wakeel, MD, ${ }^{\mathrm{a}}$ Siegfried Kropf, PhD, ${ }^{\mathrm{b}}$ Christof Stamm, MD, \\ Vladimir Alexi-Meskishvili, MD, ${ }^{\mathrm{d}}$ Felix Berger, MD, ${ }^{\mathrm{e}}$ and Titus Kuehne, MD ${ }^{\mathrm{a}, \mathrm{e}}$
}

Objective: To analyze the impact of surgery and pericardial integrity on right atrial function and total heart volume variation in the setting of pulmonary valve insufficiency.

\begin{abstract}
Methods: Right atrial function and total heart volume variation were analyzed in 2 subgroups of patients with pulmonary valve insufficiency compared with healthy controls: group 1 with surgically repaired tetralogy of Fallot ( $\mathrm{n}=20$ patients) and group 2 after balloon angioplasty of pulmonary valve stenosis in patients with isolated valve disease without surgery ( $\mathrm{n}=7$ patients). Volumetric analysis of magnetic resonance imaging data revealed parameters of atrial function (reservoir, conduit, and pump functions and cyclic volume change) and of total heart volume (end-diastolic and end-systolic total heart volume and the variation). Statistical analysis included uncorrected and corrected pairwise comparisons and the calculation of groupwise Pearson correlation coefficients.
\end{abstract}

Results: In group 1 with a pulmonary regurgitation fraction of $31.0 \% \pm 14.9 \%$, right atrial function was clearly impaired, with reduced reservoir and elevated conduit function, and total heart volume variation was elevated to $13.9 \% \pm 3.4 \%$. In group 2 with a pulmonary regurgitation fraction of $22.8 \% \pm 6.9 \%$, the values were close to normal, with unaffected atrial function and a total heart volume variation of $9.9 \% \pm 3.3 \%$.

Conclusions: The hydrodynamic effect of pulmonary valve insufficiency alone is likely not the only reason for impaired right atrial function and elevated total heart volume variation in patients with tetralogy of Fallot; it is rather the scar in the right atrium, the injured pericardium, and the disease itself that are responsible for the energetically unfavorable alterations. (J Thorac Cardiovasc Surg 2014;147:1306-11)

Pulmonary valve disease, presenting as regurgitation and/or stenosis, affect right ventricular function in many settings of congenital heart disease. Because the optimal timing for pulmonary valve replacement in pulmonary valve insufficiency in the asymptomatic patient with tetralogy of Fallot (TOF) is still not defined, ${ }^{1}$ studies with detailed analysis of right and left ventricular characteristics and ventricular interaction, ${ }^{2-4}$ as well as atrial function and atrioventricular interaction, ${ }^{5}$ are undertaken for a better risk stratification, because heart failure and life-threatening arrhythmias are dreaded. ${ }^{6}$

In our previous work, we detected impaired right atrial function in patients with pulmonary valve insufficiency

\footnotetext{
From the Departments of Congenital Heart Disease and Pediatric Cardiology, Cardiothoracic and Vascular Surgery, ${ }^{\mathrm{c}}$ and Congenital and Pediatric Cardiac Surgery, ${ }^{\mathrm{d}}$ Deutsches Herzzentrum Berlin, Berlin, Germany; the Institute of Biometry and Medical Informatics, ${ }^{\mathrm{b}}$ University of Magdeburg, Magdeburg, Germany; and the Department of Pediatric Cardiology, ${ }^{\mathrm{e}}$ Charité Universitaetsmedizin Berlin, Berlin, Germany.

This study was funded in part by the German Competence Network for Congenital Heart Defects (grant 01G10210).

Disclosures: Authors have nothing to disclose with regard to commercial support.

Received for publication Dec 20, 2012; revisions received May 29, 2013; accepted for publication June 14, 2013; available ahead of print July 29, 2013.

Address for reprints: Titus Kuehne, MD, Unit of Cardiovascular Imaging, Department of Congenital Heart Disease and Pediatric Cardiology, Deutsches Herzzentrum Berlin, Augustenburger Platz 1, D-13353 Berlin, Germany (E-mail: kuehne@dhzb.de).

0022-5223/\$36.00

Copyright $(C) 2014$ by The American Association for Thoracic Surgery

http://dx.doi.org/10.1016/j.jtcvs.2013.06.020
}

after surgical repair of TOF, with only moderate systolic and diastolic right ventricular dysfunction. ${ }^{5}$ This was reproduced by Luijnenburg and colleagues, ${ }^{7}$ who described associated impaired clinical conditions. Overall, data on detailed analysis of atrial function in congenital heart disease are sparse. Regular atrial function ${ }^{8,9}$ and a constant total heart volume $e^{10}$ have a positive impact on global cardiac output and are reported to be favorable energetically. Total heart volume variation is closely related to atrial function. Volume variations of the content of the pericardial sac occur throughout the cardiac cycle, because, in early ventricular systole, ventricular outflow is greater than atrial filling and composes $5 \%$ to $8 \%$, with a notable interindividual variation. ${ }^{10,11}$

In this study, we sought to evaluate the potential influence of surgery and pericardial integrity on right atrial function and total heart volume variation in the setting of pulmonary regurgitation. Therefore, healthy controls were compared with patients with pulmonary valve insufficiency after surgical repair of TOF and a group of patients with pulmonary valve insufficiency after balloon angioplasty of isolated pulmonary valve stenosis without preceding or subsequent surgery.

\section{METHODS}

\section{Study Population}

Twenty asymptomatic patients with repaired TOF, 7 asymptomatic patients with pulmonary valve insufficiency after balloon angioplasty of 


$$
\begin{aligned}
& \text { Abbreviations and Acronyms } \\
& \begin{aligned}
\text { ANCOVA } & =\text { analysis of covariance } \\
\text { MRI } & =\text { magnetic resonance imaging } \\
\text { THV-ED } & =\text { end-diastolic total heart volume } \\
\text { THV-ES } & =\text { end-systolic total heart volume } \\
\text { THVV } & =\text { total heart volume variation } \\
\text { TOF } & =\text { tetralogy of Fallot }
\end{aligned}
\end{aligned}
$$

pulmonary valve stenosis, and 7 healthy controls without cardiac medical history were included in the study. The patients with repaired TOF and healthy controls had been studied previously, ${ }^{5}$ after random selection from the database of the German Competence Network for Congenital Heart Defects (http://www.kompetenznetz-ahf.de) from 1 center. Patients with pulmonary valve insufficiency after pulmonary valve stenosis were recruited from routine magnetic resonance imaging (MRI) at the same center between August 2009 and April 2012. Exclusion criteria for all patients were as follows: existence of mitral or aortic valve insufficiency and tricuspid valve insufficiency greater than grade 1 in echocardiographic studies; and stenosis ( $>55 \mathrm{~mm} \mathrm{Hg}$ ) of the pulmonary outflow and arteries, assessed morphologically and reflected by elevated right ventricular peak systolic pressure, as calculated by echocardiography via tricuspid regurgitation. Sinus rhythm in the electrocardiogram was mandatory. Surgical records of patients with TOF were reviewed where available, with special regard to opening of the right atrium and fate of the pericardium at the end of the operation. The study was approved by the institutional research ethics committee, and written informed consent was obtained from the participants or their guardians.

\section{Magnetic Resonance Imaging}

Imaging of patients with TOF and healthy controls was done within the framework of the German Competence Network for Congenital Heart Defects (http://www.kompetenznetz-ahf.de). Patients with pulmonary valve insufficiency after balloon angioplasty of pulmonary valve stenosis were imaged with the same protocol. For volumetric analysis, a stack of cine images in axial orientation was acquired at end expiration, covering the whole heart. ${ }^{12}$ Slice thickness was $6 \mathrm{~mm}$ with 0 gap, and the spatial resolution was $1.4 \times 1.4 \times 6 \mathrm{~mm}^{3}$. The mean temporal resolution of 33 milliseconds, depending on the heart rate, was achieved with 25 phases per cardiac cycle.

Blood flow was measured in the pulmonary trunk with through-plane, velocity-encoded, cine MRI. ${ }^{12}$ Sequence parameters were as follows: repetition time/echo time, 5.1/3.0 milliseconds; flip angle, $15^{\circ}$; and encoding velocity, 200 to $300 \mathrm{~cm} / \mathrm{s}$.

\section{Image Analysis}

For image analysis, the software View Forum, release 6.3 V1L7 SP1 (Philips Medical Systems, Irvine, Calif) was used. Blood flow measurements revealed effective stroke volumes and regurgitation fractions. For volumetric analysis of the right ventricle, ${ }^{12}$ the right atrium, ${ }^{5,13}$ and the total heart, contours were manually traced in every slice of the stack of axial cine MR images using the Simpson rule. The atrial appendages were included in the measurement of atrial volumes; superior and inferior caval veins, coronary sinus, and pulmonary veins were excluded at their junction to the atrium. ${ }^{5}$

\section{Right Ventricular Function}

End-diastolic volume, end-systolic volume, and ejection fraction were determined. ${ }^{12}$ Volumes were indexed by dividing them by the body surface area.

\section{Right Atrial Function}

For a detailed analysis of atrial function, the following parameters were measured: maximal volume at ventricular end systole, minimal volume at ventricular end diastole, and cyclic volume change by subtraction of minimal from maximal volume, all indexed to the body surface area. ${ }^{13}$ Dividing the cyclic volume change by the maximal atrial volume, multiplied by 100 , resulted in the total atrial filling fraction.

Reservoir volume was calculated by subtracting minimal atrial volume at mid diastole from maximal atrial volume (Figure 1).5 Pump volume was calculated by subtracting minimal atrial volume from maximal atrial volume at mid diastole. Conduit volume resulted after subtraction of the sum of reservoir and pump volume from the effective stroke volume, which was quantified by flow measurement in the pulmonary trunk. Reservoir, conduit, and pump functions were expressed as a percentage of the effective stroke volume. The terms systole and diastole always refer to ventricular systole and ventricular diastole, respectively.

\section{Total Heart Volume Variation}

To measure the entire content of the pericardial sac, contours were manually traced in each slice of the stack at ventricular end diastole and end systole, along the pericardial border (Figure 2), which revealed end-diastolic and end-systolic total heart volumes (THV-ED and THV$\mathrm{ES}$, respectively) in $\mathrm{mL}$. The volumes were indexed to the body surface area. Total heart volume variation (THVV), in percentage, was calculated according to the following formula:

$\operatorname{THVV}[\%]=(\mathrm{THV}-\mathrm{ED}[\mathrm{mL}]-\mathrm{THV}-\mathrm{ES}[\mathrm{mL}]) / \mathrm{THV}-\mathrm{ED}[\mathrm{mL}] \times 100$.

\section{Statistical Analysis}

All parameters of ventricular and atrial size and function and total heart volume are reported as means and SDs. Because graphical analysis of parameters showed an approximately gaussian distribution, a 1-way analysis of covariance (ANCOVA) of all 3 groups was performed. By using arguments of closed testing procedures, the familywise type I error for the pairwise comparisons was maintained by performing unadjusted paired tests (least significant difference) and combining the resulting $P$ values with that of the global ANCOVA $P$ value. The final $P$ value was the maximum of the pairwise and global $P$ value. These computations were done with SPSS 17.0 (SPSS, Inc, Chicago, Ill).

To account for possible differences in important patient characteristics among the 3 patient groups, all computations were repeated with pulmonary regurgitation and body surface area as corrective covariables (ANCOVA). In cases of significant effects of the covariables, these were considered in detail by the calculation of groupwise Pearson correlation coefficients between the covariables and the target variable. SAS 9.2 (SAS Institute, Inc, Cary, NC) was used for the latter testing.

Pearson correlation was also computed for total heart volume variation and right atrial conduit function.

\section{RESULTS Patient Characteristics}

Twenty patients with repaired TOF (aged $19.5 \pm 8.9$ years; body surface area, $1.6 \pm 0.3 \mathrm{~m}^{2} ; 8$ men and 12 women), 7 with pulmonary valve insufficiency after balloon angioplasty of pulmonary valve stenosis (aged $14.0 \pm 4.2$ years; body surface area, $1.4 \pm 0.4 \mathrm{~m}^{2} ; 3$ men and 4 women), in the following also entitled as "pulmonary stenosis patients," and 7 healthy controls (aged $24.6 \pm 8.7$ years; 


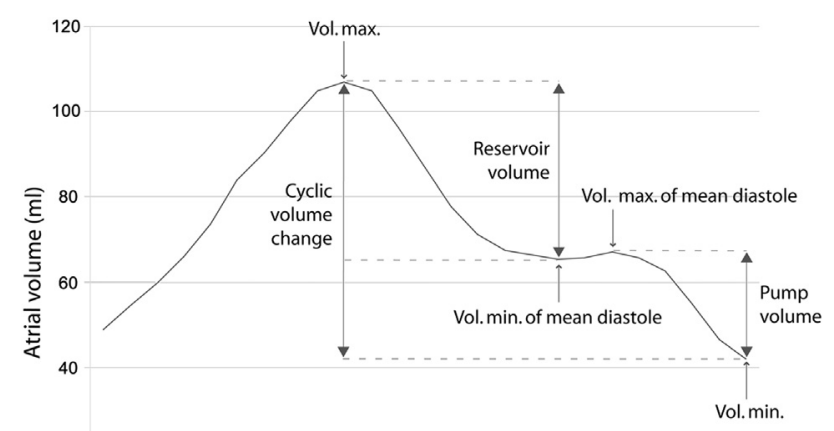

20 Conduit volume $=$ eff. stroke volume $-($ reservoir + pump volume $)$

0

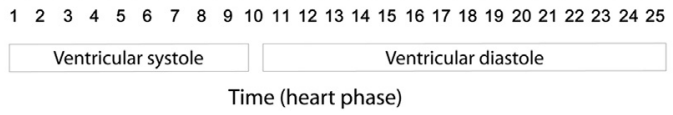

FIGURE 1. Atrial function. Shown is an exemplary atrial time volume curve of a healthy control with assessment of parameters of atrial function. Although reservoir, pump, and cyclic volume changes are calculated from atrial volumes at special time points (see Methods), the conduit volume is calculated by the subtraction of the sum of reservoir and pump volume from the effective stroke volume of the right ventricle, measured as effective forward flow in the pulmonary trunk. Vol. max., Maximum volume; Vol. min., minimum volume.

body surface area, $1.9 \pm 0.3 \mathrm{~m}^{2} ; 4$ men and 3 women) without cardiac medical history were enrolled in the study.

The right ventricular systolic pressure, as assessed via mild tricuspid valve insufficiency by echocardiography, was slightly elevated to $40 \pm 14 \mathrm{~mm} \mathrm{Hg}$ in the patients with TOF and to $31 \pm 3 \mathrm{~mm} \mathrm{Hg}$ in the patients with pulmonary stenosis.

Patients' age at balloon valvuloplasty of the pulmonary valve ranged from 5 days to 6 years $(1.5 \pm 2.5$ years). One infant had repeated interventions.

Ten of the patients with TOF had palliations before corrective surgery. Corrective surgery was performed with transannular patch $(\mathrm{n}=9)$, without transannular patch $(n=2)$, or with valved conduit insertion $(n=9)$. Four patients were corrected late, with an age ranging from
11 to 23 years, and the other 16 patients were $2.0 \pm 1.3$ years old at primary correction. Four patients had repeated surgery, and at the time of MRI, 9 had a homograft or heterograft.

All patients whose operation records were available had atrial opening during surgery, the incision being described as marginal in $23 \%$, diagonal in $31 \%$, transverse in $15 \%$, and without specification in $31 \%$. Pericardial integrity was restored ("loosely adapted") with the use of a surgical membrane in $62 \%$ of patients, and there was no statement about the fate of the pericardium in the remaining $38 \%$. In 1 patient, a pericardectomie was documented with unknown reason 3 months after corrective surgery.

\section{Right Ventricular Function and Pulmonary Regurgitation}

Right ventricular volumes were enlarged in both patient groups compared with healthy controls, caused by pulmonary regurgitation, which was slightly higher in the patients with TOF $(31.0 \% \pm 14.9 \%)$ compared with the patients with pulmonary stenosis $(22.8 \% \pm 6.9 \% ; P=.1287)$.

Results of right ventricular volumetric analysis are listed in Table 1. Results of statistical testing are specified in Table 2.

\section{Right Atrial Function}

A comparison of maximal and minimal volumes of the right atrium revealed minor differences between the 3 groups (Table 1 ). The cyclic volume change was clearly reduced in patients with TOF $(P<.0001)$ and slightly reduced in patients with pulmonary stenosis $(P=.3681)$ compared with healthy controls. Patients with TOF had abnormal values for all parameters of atrial function, whereas in the patients with pulmonary valve stenosis, atrial function was nearly normal (Tables 1 and 2).

Corrected for pulmonary regurgitation, the results were unaffected, as specified in Table 2. When corrected for pulmonary regurgitation and body surface area, the results were similar.
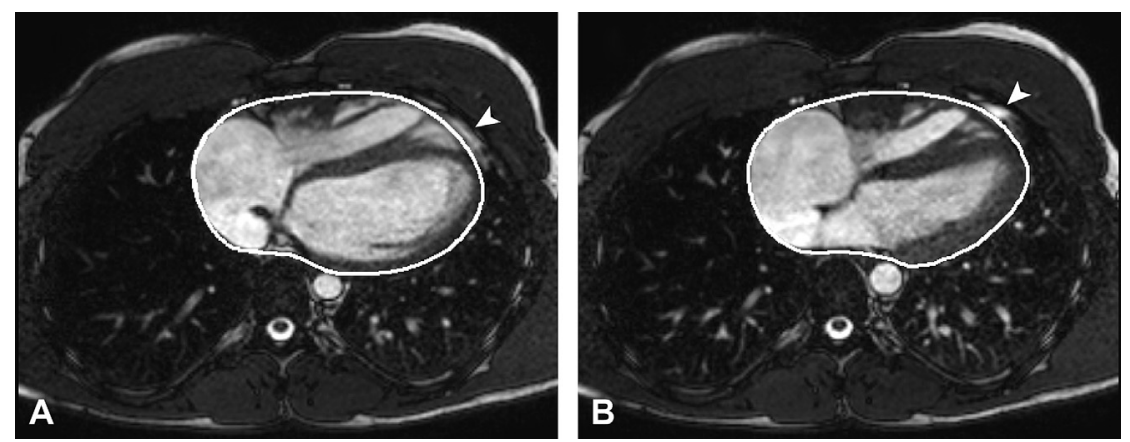

FIGURE 2. Tracing of the pericardial contours on the transversal cine images in ventricular diastole (A) and systole (B) in a patient after repair of tetralogy of Fallot. Extrapericardial fat was excluded (arrowhead). 
TABLE 1. Parameters of right ventricle, right atrium, and total heart

\begin{tabular}{lccc}
\hline \multicolumn{1}{c}{ Parameter } & TOF patients & PS patients & $\begin{array}{c}\text { Healthy } \\
\text { controls }\end{array}$ \\
\hline Right ventricle & & & \\
RV-EDV, $\mathrm{mL} / \mathrm{m}^{2}$ & $135.2 \pm 27.9$ & $112.0 \pm 38.0$ & $102.0 \pm 17.4$ \\
RV-EF, \% & $48.0 \pm 4.1$ & $61.5 \pm 5.4$ & $55.6 \pm 4.2$ \\
Right atrium & & & \\
RA vol. max., $\mathrm{mL} / \mathrm{m}^{2}$ & $50.8 \pm 17.1$ & $59.3 \pm 10.9$ & $57.3 \pm 9.3$ \\
RA vol. min., $\mathrm{mL} / \mathrm{m}^{2}$ & $29.8 \pm 11.7$ & $27.3 \pm 9.8$ & $22.5 \pm 4.9$ \\
Cycl. vol. change, $\mathrm{mL} / \mathrm{m}^{2}$ & $21.1 \pm 6.5$ & $32.0 \pm 4.1$ & $34.9 \pm 5.4$ \\
Filling fraction, \% & $42.3 \pm 8.2$ & $55.0 \pm 8.1$ & $61.0 \pm 4.2$ \\
Reservoir function, \% & $26.1 \pm 9.2$ & $42.7 \pm 10.4$ & $41.0 \pm 3.4$ \\
Conduit function, \% & $56.2 \pm 12.6$ & $26.9 \pm 13.3$ & $35.2 \pm 5.5$ \\
Pump function, \% & $17.8 \pm 6.0$ & $30.2 \pm 9.1$ & $23.9 \pm 6.3$ \\
Total heart & & & \\
TH-EDV, $\mathrm{mL} / \mathrm{m}^{2}$ & $439.0 \pm 68.9$ & $367.2 \pm 57.9$ & $387.8 \pm 35.6$ \\
TH-ESV, $\mathrm{mL} / \mathrm{m}^{2}$ & $378.3 \pm 65.2$ & $330.2 \pm 46.5$ & $357.3 \pm 32.2$ \\
THVV, \% & $13.9 \pm 3.4$ & $9.9 \pm 3.3$ & $7.8 \pm 2.3$ \\
\hline
\end{tabular}

Values are means \pm SD. TOF, Tetralogy of Fallot; $P S$, primary pulmonary stenosis; $R V$ - $E D V$, right ventricular end-diastolic volume; $R V-E F$, right ventricular ejection fraction; $R A$ vol. max., maximal right atrial volume; $R A$ vol. min., minimal right atrial volume; Cycl. vol., cyclic volume; TH-EDV, end-diastolic total heart volume; $T H$-ESV, end-systolic total heart volume; THVV, total heart volume variation.

With elevated pulmonary regurgitation, reservoir function was higher (Pearson correlation, 0.637; $P=.003$ ) and conduit function lower (Pearson correlation, -0.650 ; $P=.002)$ in the patients with TOF, whereas in the patients with pulmonary valve stenosis, maximal volume (Pearson correlation, $-0.762 ; P=.047)$ and cyclic volume change (Pearson correlation, $-0.868 ; P=.011$ ) were lower.

\section{Total Heart Volume Variation}

The total heart volume variation was notably higher in patients with TOF and slightly elevated in patients with pulmonary valve stenosis, compared with healthy controls (Table 1). The patient with documented pericardectomy had the highest total heart volume variation $(20.3 \%)$.

Comparing the 2 patient groups, the values of the pulmonary valve stenosis group were closer to normal. The results were unaffected by pulmonary regurgitation and body surface area, as tested by analysis of covariance. The mean difference of total heart volume variation between patients with TOF and healthy controls was 7.6\% (95\% confidence interval, $3.1 \%-11.4 \%$ ), when corrected for pulmonary regurgitation and body surface area.

There was a positive correlation between total heart volume variation and right atrial conduit function in both patient groups (Pearson correlation of $0.472[P=.036]$ in patients with TOF and Pearson correlation of 0.853 $[P=.015]$ in patients with pulmonary stenosis).

\section{DISCUSSION}

The analysis of right atrial function and total heart volume variation in the setting of pulmonary regurgitation, with and without previous surgery, reveals major differences. In the studied patients with TOF and pulmonary regurgitation after surgical repair, atrial dysfunction and a considerably elevated total heart volume variation are present. Both changes are disadvantageous energetically. 8,10 In contrast, in patients with pulmonary valve insufficiency

TABLE 2. Results of statistical testing ( $P$ values)

\begin{tabular}{|c|c|c|c|c|c|c|c|c|c|}
\hline \multirow[b]{2}{*}{ Parameter } & \multirow{2}{*}{$\begin{array}{c}\text { Uncorrected } \\
\text { ANOVA } \\
\text { All } 3 \text { groups }\end{array}$} & \multicolumn{3}{|c|}{$\begin{array}{c}\text { Uncorrected pairwise } \\
\text { comparison }\end{array}$} & \multicolumn{2}{|c|}{$\begin{array}{c}\text { For PR } \\
\text { corrected ANCOVA }\end{array}$} & \multicolumn{2}{|c|}{$\begin{array}{l}\text { Corrected pairwise } \\
\text { comparison }\end{array}$} & \multirow{2}{*}{$\begin{array}{c}\begin{array}{c}\text { Corrected } \\
\text { pairwise }\end{array} \\
\text { comparison } \\
\text { PS vs TOF } \\
\end{array}$} \\
\hline & & C vs PS & C vs TOF & PS vs TOF & All 3 groups & PR & C vs PS & C vs TOF & \\
\hline RV-EDV & $.0236 *$ & .5156 & $.0236^{*}$ & .0738 & .2836 & .2871 & .9775 & .2836 & .2836 \\
\hline RV-EF & $<.0001 *$ & $.0174 *$ & $.0004 *$ & $<.0001 *$ & $<.0001 *$ & .3645 & .0979 & $.0016^{*}$ & $<.0001 *$ \\
\hline PR & $<.0001 *$ & $.0030 *$ & $<.0001 *$ & .1287 & NA & NA & NA & NA & NA \\
\hline RA vol. max. & .3537 & .8087 & .3537 & .3537 & .1914 & .2575 & .7174 & .1914 & .1914 \\
\hline RA vol. min. & .2867 & .3913 & .2867 & .5870 & .8623 & .3952 & .8623 & .8623 & .8623 \\
\hline Cycl. vol. change & $<.0001 *$ & .3681 & $<.0001 *$ & $.0002 *$ & $<.0001 *$ & .1797 & .1468 & $<.0001 *$ & $.0001 *$ \\
\hline Filling fraction & $<.0001 *$ & .1440 & $<.0001 *$ & $.0006 *$ & $.0002 *$ & .7280 & .1561 & $.0002 *$ & $.0008 *$ \\
\hline Reservoir function & $.0001 *$ & .7093 & $.0004 *$ & $.0001 *$ & $<.0001 *$ & $.0014 *$ & .1756 & $<.0001 *$ & $<.0001 *$ \\
\hline Conduit function & $<.0001 *$ & .1973 & $.0003^{*}$ & $<.0001 *$ & $<.0001 *$ & $.0016 *$ & .6878 & $<.0001 *$ & $<.0001 *$ \\
\hline Pump function & $.0006 *$ & .0805 & $.0483 *$ & $.0006^{*}$ & $.0004 *$ & .1962 & .3642 & $.0223 *$ & $.0004 *$ \\
\hline TH-EDV & $.0227 *$ & .5376 & .0680 & $.0227 *$ & $.0161 *$ & .2786 & .9914 & $.0418^{*}$ & $.0463 *$ \\
\hline TH-ESV & .1623 & .3793 & .4040 & .1623 & .1211 & .4056 & .7316 & .2426 & .1211 \\
\hline THVV & $.0002 *$ & .2414 & $.0002 *$ & $.0073 *$ & $.0010 *$ & .3212 & .1342 & $.0010^{*}$ & $.0049 *$ \\
\hline BSA & $.0279 *$ & $.0279 *$ & $.0483^{*}$ & .1850 & .0778 & .8678 & .0778 & .1954 & . 1935 \\
\hline
\end{tabular}

Corrected pairwise comparison indicates corrected for pulmonary regurgitation; for detailed description of the statistical methods used, see Methods. ANOVA, Analysis of variance; $A N C O V A$, analysis of covariance; $C$, healthy controls; $P S$, primary pulmonary stenosis; $T O F$, tetralogy of Fallot; $P R$, pulmonary regurgitation; $R V$-EDV, right ventricular end-diastolic volume; $R V-E F$, right ventricular ejection fraction; $N A$, not applicable; $R A$ vol. max., maximal right atrial volume; $R A$ vol min., minimal right atrial volume; $C y c l$. vol., cyclic volume; $T H-E D V$, end-diastolic total heart volume; $T H$-ESV, end-systolic total heart volume; $T H V V$, total heart volume variation; $B S A$, body surface area. * Significant differences are in bold. 
after balloon angioplasty of isolated valve stenosis, the parameters of atrial function and total heart volume variation are close to normal. Thus, the hydrodynamic effect of pulmonary regurgitation alone does not seem to be the leading cause for the observed changes in patients with TOF.

Variations of the total heart volume during the cardiac cycle have been described before in the healthy group ${ }^{10,11}$ and match with the presented results in the control group (approximately $8 \%$ ). Concerning energy expenditure, in theory, a low total heart volume variation is most efficient, because the surrounding structures have to be moved to a lesser extent. To save energy, the healthy heart has several features to keep the volume inside the pericardial sac constant during the cardiac cycle. The atria, especially the atrial appendages, acts as a reservoir during ventricular systole and fills out the space resulting from ventricular contraction. ${ }^{10,14}$ Hence, unimpaired function of both atria, especially their reservoir function, plays an important role in total heart volume constancy. When comparing right with left atrial function, the right atrium has a higher reservoir and the left atrium a higher conduit function, ${ }^{5,15}$ which can be explained by the more appendage-like structure of the right atrium in its entirety. ${ }^{16}$ The right atrium plays a crucial role in total heart volume constancy. If the reservoir function of the atria during rapid emptying of the ventricles in systole is reduced, as is the case in the right atrium in the studied patients with TOF, a higher right atrial conduit flow is necessary to fill the right ventricle adequately during diastole. ${ }^{5}$ The conduit volume passes the atrium without changing its size and fills the ventricle directly, somewhat like a continuous venous flow return. It compensates for volume reduction during rapid ventricular emptying in systole, which is not compensated by atrial reservoir capacity, and generates the volume variation of the heart during the cardiac cycle. This has been demonstrated for the left side of the heart by Bowman and Kovacs $^{17}$ : the higher the conduit flow, the higher the total heart volume variation. The present study verifies this for the right side of the heart, because there is a strong positive correlation between right atrial conduit function and total heart volume variation. The elevation of conduit function has been associated with reduced cardiac output. ${ }^{8}$

Normal pericardial function is important for regular atrial function as well, ${ }^{8}$ and also seems to play a role in the maintenance of a constant heart volume. In patients before and after coronary bypass surgery, in whom the pericardium was loosely opposed by 2 to 4 stitches, but not closed, the total heart volume variation was slightly, but significantly, elevated from $6.9 \% \pm 0.5 \%$ to $7.5 \% \pm 0.4 \%$ after surgery. ${ }^{18}$ In 1 patient with absent pericardium, total heart volume variation was elevated to $12 \%{ }^{19}$; in 1 of our patients with TOF, it was elevated even to $20.3 \%$ after pericardectomy. The inserted surgical membrane or absent pericardial integrity in our patient group with TOF after surgery could contribute to the augmented total heart volume variation and also to atrial dysfunction. Atrial dysfunction can be caused by surgery as well, because the right atrium is usually opened in patients for TOF correction. The negative influence of a pericardiotomy on left atrial function has been shown in a porcine model, where reservoir and pump functions were reduced, independently of an additional Cox maze procedure. ${ }^{20}$ On the other hand, a procedure named pericardial meshing, which means 20 to 30 longitudinal small incisions by scalpel, was associated with an increase of left ventricular chamber compliance and cardiac output in dogs. ${ }^{21}$ However, atrial function and total heart volume variation were not assessed in this study.

With the present study, it is shown that right atrial dysfunction in patients with $\mathrm{TOF}^{5,22}$ is associated with an elevated total heart volume variation. In the studied patients with a pure pulmonary valve problem, the otherwise healthy heart seems to be able to compensate for pulmonary reverse flow, because parameters of atrial function and total heart volume variation are the same as in the studied healthy controls. The mechanism could be the elevated pump function of the right atrium, albeit this elevation is without statistical significance, and a higher filling of the right ventricle in early diastole. In the studied patients with TOF, ventricular filling is not elevated, ${ }^{5}$ and atrial pump function is even reduced. The energetically unfavorable conduit flow is elevated as a mechanism of compensation, as previously described.

The development of arrhythmias is a well-known problem in patients with TOF, and, in addition to other factors, atrial enlargement and repeated cardiac surgical procedures induce atrial arrhythmias. ${ }^{23}$ Zeltser and colleagues ${ }^{24}$ showed, in an animal model, that right ventricular volume overload is also responsible for atrial arrhythmias. In our studied patients with TOF, right atria were not enlarged, and all patients were in sinus rhythm. Further studies are warranted, because we cannot conclude from the presented findings if the observed changes in atrial function might evoke late rhythm problems.

Limitations of this study are the few patients with pulmonary stenosis and their younger age and lower body surface area. However, the corrected statistical testing for the latter parameter did not affect the results. The comparison of patients with TOF with patients with pulmonary stenosis is somewhat disadvantageous because 2 different pathologic entities are compared. However, for assessment of the hydrodynamic effects of pulmonary regurgitation, it is an acceptable compromise. The natural history of patients with isolated congenital pulmonary valve incompetence, studied nearly 30 years ago, has led to the consideration of inserting a pulmonary valve in patients with TOF with pulmonary regurgitation and increasing cardiomegaly. ${ }^{25}$ 
The energy loss due to the elevated total heart volume variation and reduced atrial reservoir function is not quantified, which should be a subject for future research.

In conclusion, right atrial function and total heart volume variation can be normal in the presence of pulmonary regurgitation, as shown in the patients with primary pulmonary valve stenosis. Hence, the hydrodynamic effects of pulmonary valve insufficiency alone are likely not the only reasons for atrial dysfunction and elevated total heart volume variation in the studied patients with TOF. It is rather the scar in the right atrium, the injured pericardium, and the disease itself that are responsible for the observed energetically unfavorable alterations.

\section{References}

1. Burchill LJ, Wald RM, Harris L, Colman JM, Silversides CK. Pulmonary valve replacement in adults with repaired tetralogy of Fallot. Semin Thorac Cardiovasc Surg Pediatr Card Surg Annu. 2011;14:92-7.

2. Diller GP, Kempny A, Liodakis E, Alonso-Gonzalez R, Inuzuka R, Uebing A, et al. Left ventricular longitudinal function predicts life-threatening ventricular arrhythmia and death in adults with repaired tetralogy of Fallot. Circulation. 2012;125:2440-6

3. Mueller M, Rentzsch A, Hoetzer K, Raedle-Hurst T, Boettler P, Stiller B, et al. Assessment of interventricular and right-intraventricular dyssynchrony in patients with surgically repaired tetralogy of Fallot by two-dimensional speckle tracking. Eur J Echocardiogr. 2010;11:786-92.

4. Parish V, Valverde I, Kutty S, Head C, Qureshi SA, Sarikouch S, et al. Dobutamine stress MRI in repaired tetralogy of Fallot with chronic pulmonary regurgitation: a comparison with healthy volunteers. Int J Cardiol. 2013;166:96-105.

5. Riesenkampff E, Mengelkamp L, Mueller M, Kropf S, Abdul-Khaliq H, Sarikouch S, et al. Integrated analysis of atrioventricular interactions in tetralogy of Fallot. Am J Physiol Heart Circ Physiol. 2010;299:H364-71.

6. Gatzoulis MA, Balaji S, Webber SA, Siu SC, Hokanson JS, Poile C, et al. Risk factors for arrhythmia and sudden cardiac death late after repair of tetralogy of Fallot: a multicentre study. Lancet. 2000;356:975-81.

7. Luijnenburg SE, Peters RE, van der Geest RJ, Moelker A, Roos-Hesselink JW, de Rijke YB, et al. Abnormal right atrial and right ventricular diastolic function relate to impaired clinical condition in patients operated for tetralogy of Fallot [published online ahead of print March 3, 2012]. Int J Cardiol. doi: 10.1016/j.ij card.2012.02.011.

8. Gaynor SL, Maniar HS, Prasad SM, Steendijk P, Moon MR. Reservoir and conduit function of right atrium: impact on right ventricular filling and cardiac output. Am J Physiol Heart Circ Physiol. 2005;288:H2140-5.

9. Guyton RA, Andrews MJ, Hickey PR, Michaelis LL, Morrow AG. The contribution of atrial contraction to right heart function before and after right ventriculotomy: experimental and clinical observations. J Thorac Cardiovasc Surg. 1976;71:1-10.
10. Carlsson M, Cain P, Holmqvist C, Stahlberg F, Lundback S, Arheden H. Total heart volume variation throughout the cardiac cycle in humans. Am J Physiol Heart Circ Physiol. 2004;287:H243-50.

11. Bowman AW, Kovacs SJ. Assessment and consequences of the constant-volume attribute of the four-chambered heart. Am J Physiol Heart Circ Physiol. 2003; 285:H2027-33.

12. Sarikouch S, Koerperich H, Dubowy KO, Boethig D, Boettler P, Mir TS, et al. Impact of gender and age on cardiovascular function late after repair of tetralogy of Fallot: percentiles based on cardiac magnetic resonance. Circulation. 2011;4: 703-11.

13. Sarikouch S, Koerperich H, Boethig D, Peters B, Lotz J, Gutberlet M, et al Reference values for atrial size and function in children and young adults by cardiac MR: a study of the German competence network congenital heart defects. J Magn Reson Imaging. 2011;33:1028-39.

14. Carlsson M, Ugander M, Heiberg E, Arheden H. The quantitative relationship between longitudinal and radial function in left, right, and total heart pumping in humans. Am J Physiol Heart Circ Physiol. 2007;293:H636-44.

15. Jarvinen VM, Kupari MM, Hekali PE, Poutanen VP. Right atrial MR imaging studies of cadaveric atrial casts and comparison with right and left atrial volumes and function in healthy subjects. Radiology. 1994;191:137-42.

16. Anderson RH, Cook AC. The structure and components of the atrial chambers. Europace. 2007;9(suppl 6):vi3-9.

17. Bowman AW, Kovacs SJ. Left atrial conduit volume is generated by deviation from the constant-volume state of the left heart: a combined MRI-echocardiographic study. Am J Physiol Heart Circ Physiol. 2004;286: H2416-24.

18. Carlsson M, Rosengren A, Ugander M, Ekelund U, Cain PA, Arheden H. Center of volume and total heart volume variation in healthy subjects and patients before and after coronary bypass surgery. Clin Physiol Funct Imaging. 2005;25:226-33.

19. Waters EA, Bowman AW, Kovacs SJ. MRI-determined left ventricular "crescent effect": a consequence of the slight deviation of contents of the pericardial sack from the constant-volume state. Am J Physiol Heart Circ Physiol. 2005;288:H848-53.

20. Voeller RK, Zierer A, Lall SC, Sakamoto S, Chang NL, Schuessler RB, et al. The effects of the Cox maze procedure on atrial function. J Thorac Cardiovasc Surg. 2008;136:1257-64, e1-3.

21. Oe M, Asou T, Morita S, Fukamachi K, Mitani A, Tokunaga K. Beneficial effect of pericardial meshing on left ventricular pump performance in dogs. J Thorac Cardiovasc Surg. 1991;101:260-8.

22. Hui W, Abd El Rahman MY, Dsebissowa F, Rentzsch A, Gutberlet M Alexi-Meskishvili V, et al. Quantitative analysis of right atrial performance after surgical repair of tetralogy of Fallot. Cardiol Young. 2004;14:520-6.

23. Khairy P, Aboulhosn J, Gurvitz MZ, Opotowsky AR, Mongeon FP, Kay J, et al Arrhythmia burden in adults with surgically repaired tetralogy of Fallot: a multi-institutional study. Circulation. 2010;122:868-75.

24. Zeltser I, Gaynor JW, Petko M, Myung RJ, Birbach M, Waibel R, et al. The roles of chronic pressure and volume overload states in induction of arrhythmias: an animal model of physiologic sequelae after repair of tetralogy of Fallot. J Thorac Cardiovasc Surg. 2005;130:1542-8.

25. Shimazaki Y, Blackstone EH, Kirklin JW. The natural history of isolated congenital pulmonary valve incompetence: surgical implications. Thorac Cardiovasc Surg. 1984;32:257-9. 